\title{
Improving the region of attraction of ITER in the presence of actuator saturation
}

\author{
J-Y. Favez ${ }^{1,2}$, Ph. Mullhaupt ${ }^{1}$, B. Srinivasan ${ }^{1}$, J.B. Lister ${ }^{2}$, D. Bonvin ${ }^{1}$ \\ ${ }^{1}$ Laboratoire d'Automatique, Ecole Polytechnique Fédérale de Lausanne, \\ CH-1015 Lausanne, Switzerland \\ ${ }^{2}$ Centre de Recherches en Physique des Plasmas, Ecole Polytechnique Fédérale de Lausanne, \\ CH-1015 Lausanne, Switzerland \\ email: jean-yves.favez@epfl.ch; fax: +41 216932574
}

\begin{abstract}
The control of the ITER tokamak unstable vertical position is considered in the presence of actuator saturation. Linearised models of the ITER system all share the feature of a single unstable pole (attributable to the vertical instability) and a large number of stable poles. The aim of this work is to improve the existing controller in the sense of increasing the region of attraction while retaining the local performance around the origin. For second order systems with one unstable and one stable pole under saturated input, it has been shown that these requirements can be achieved using an additional nonlinear term in the existing linear control law. In this work, we discuss the extension to this nonlinear control law for higher order systems like the ITER tokamak. The validation of this new controller is done via simulations.
\end{abstract}

\section{INTRODUCTION}

The control of the current, position and shape of a tokamak plasma is complicated by the instability of the vertical position if the plasma cross section is elongated. Elongation of the plasma shape is a feature of all modern tokamaks, necessary to optimise the use of the magnetic field and will be used in the future ITER tokamak. Considerable work has gone into modelling the current, position and shape control of ITER, demonstrating adequate controllability with a large variety of models and controller designs. Linearised models of the system to be controlled all share the feature of a single unstable pole (attributable to the vertical instability) and a large number of stable poles (attributable to positive resistance in all other circuit equations). Existing experiments have exploited the control of vertically unstable plasmas with little difficulty.

Due to the size and therefore the cost of the ITER project, there will inevitably be smaller margins allowed in the power supplies to control the currents in the Poloidal Field coils, which are the actuators of the plasma current, position and shape feedback control system. The implication of this is that the feedback control loop may experience actuator saturation during large transients, which are frequent events due to a variety of perturbations inside the plasma itself. This saturation can be of two types, limiting the power supply voltage, or limiting the delivered electrical current.

The power supply current saturation is more benign, due to the integrating nature of the system to be controlled. The Poloidal Field coil currents cannot vary faster than the applied voltages permit, resulting in a reasonable time horizon for strategically handling the approach to such saturation. On the other hand, voltage saturation is produced by the feedback controller itself, with no intrinsic delay.

The object of this present paper is to explore the design of a feedback controller which explicitly takes into consideration the saturation of the power supply voltages when producing the power supply demand signals. In this work we only consider the vertically stabilising part of the controller (fast controller). Since for this task there is one power supply planned we consider systems with a single saturated input. The aim is to use the reference controller proposed by [2] and to enlarge its region of attraction (i.e. the region in state space from which the closed-loop system asymptotically reaches the origin [1], [5]) to the null controllable region (i.e. the region in state space where there exists an open-loop input that can steer the system to the origin [1], [5], [6], [7]).

In a former work [3] we considered a system with a single unstable pole and a single stable pole. We derived a formal definition of the region of attraction of the closed loop system with saturation of the single input and we examined the performance of this controller.

The main contributions of this work are: i) the extension of this analysis to a system with a single unstable pole and multiple stable poles and ii) the discussion of the excursion of the trajectory outside the null controllable region during a perturbation (ELM).

The paper is organised as follows. In Section II, definitions and terms used in this paper are introduced. Section III sums up the theory for second order systems. In Section IV, the controller for higher order systems is discussed. Section V compares the proposed controller with the reference controller via simulations, and conclusions are drawn in Section VI.

\section{Preliminaries}

\section{A. Closed loop ITER system}

The ITER closed loop system for controller design is composed of 3 parts (Fig. 1a)).

1) ITER tokamak:

The typical linear model of ITER consists of $50 \ldots 100$ states, 11 superconducting Poloidal Field coils (voltage inputs) and several outputs $y$. These outputs represent the vertical $z$ and radial $R$ positions of the plasma, 
the gaps $g_{1} \ldots g_{6}$ between the edge of the plasma and the surrounding plasma facing components (Fig. 2), the plasma current and the magnetic diagnostic measurements (about 100 sensors). The vertical position of the plasma $z$ is used for the vertical stabilisation. There are two supplementary inputs $w$ with which perturbations like edge localised modes (ELMs) are simulated.

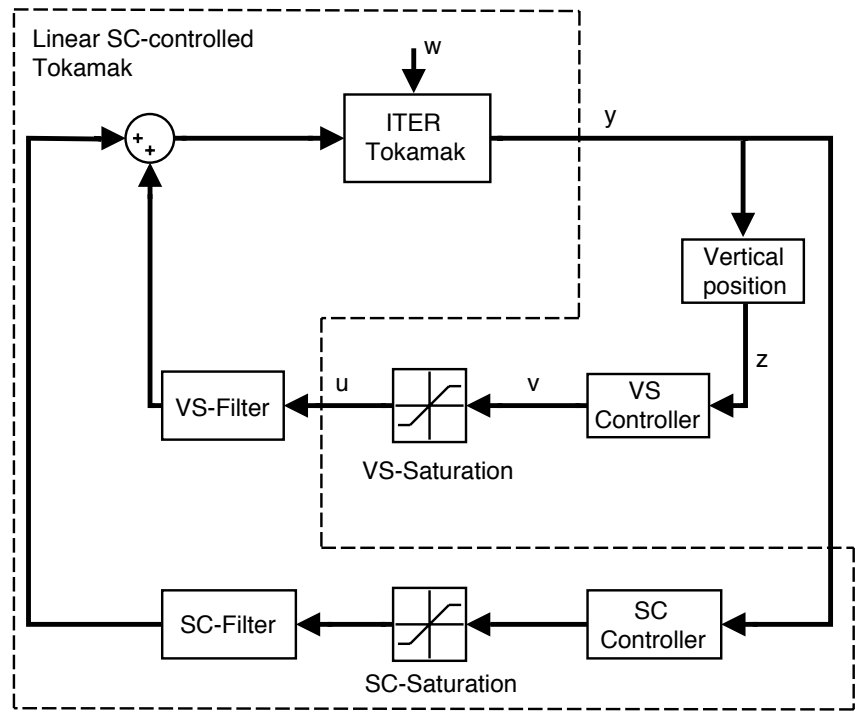

a)

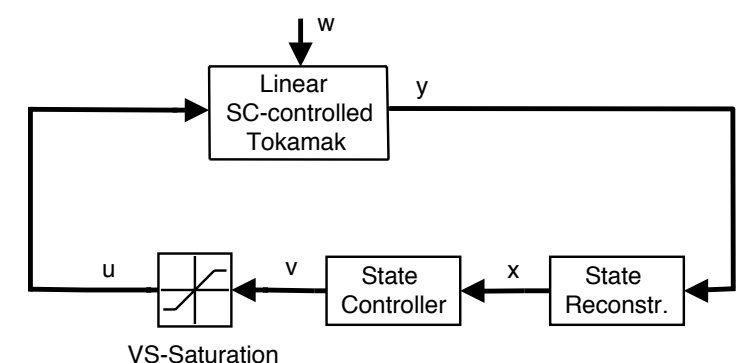

Fig. 1. Closed loop ITER system. a) Reference closed loop system. b) Linear SC-controlled tokamak with state reconstruction and linear state feedback controller.

2) Vertical stabilising controller and power supply (VS): The controller is a simple PD controller [2].

A single power supply drives current in 4 of the 11 coils. The power supply is modeled by a saturation and a low-pass filter.

3) Plasma shape controller and power supplies (SC):

The controller was designed by [2]. It is a low order controller which is mainly based on the singular perturbation method. Thus, it only controls the slowest modes of the system which are due to the 11 superconducting coils.

For plasma shape control, each coil of the tokamak is actuated by one power supply. Every power supply is modeled by a saturation and a low-pass filter.

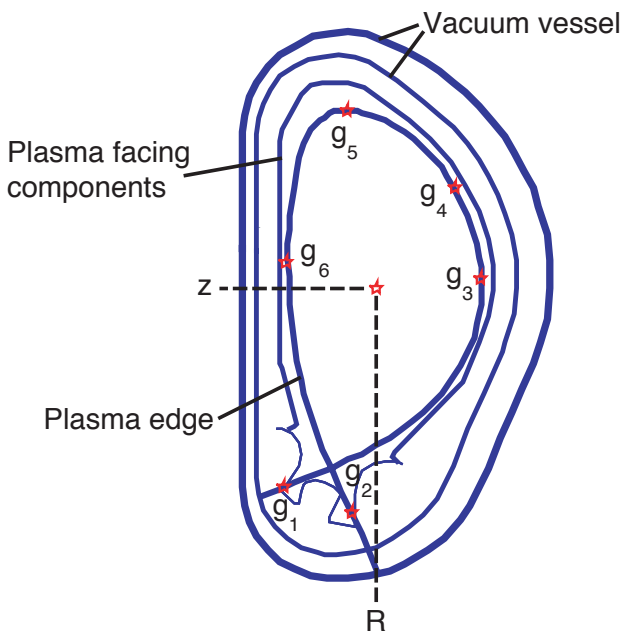

Fig. 2. Definition of the vertical $z$ and radial $R$ plasma position and the gaps $g_{1} \ldots g_{6}$

For our purposes we assume that the SC-controller never saturates the power supplies. Thus, we combine the tokamak, both low-pass filters and the SC-controller (blocks enclosed by the dashed frame in Fig. 1a)) into a linear single system (linear SC-controlled tokamak) by expressing it as a state space model

$$
\begin{gathered}
\dot{x}=A x+E \dot{w}+b u \\
y=C x
\end{gathered}
$$

where, $x \in \mathbb{R}^{n}$ is the state vector, $u \in \mathbb{R}$ the input and $\dot{w} \in \mathbb{R}^{l}$ the time derivative of the ELM perturbation. Without loss of generality, $A$ can be written as

$$
A=\left[\begin{array}{ccccccc}
\lambda_{1} & 0 & \cdots & 0 & 0 & \cdots & 0 \\
0 & \lambda_{2} & \cdots & 0 & 0 & \cdots & 0 \\
\vdots & \vdots & \ddots & \vdots & \vdots & \vdots & \vdots \\
0 & 0 & \cdots & \lambda_{r} & \mu_{r} \nu_{r} & \cdots & 0 \\
0 & 0 & \cdots & -\mu_{r} / \nu_{r} & \lambda_{r} & \cdots & 0 \\
\vdots & \vdots & \vdots & \vdots & \vdots & \ddots & \vdots \\
0 & 0 & \cdots & 0 & 0 & \cdots & \lambda_{m}
\end{array}\right]
$$

where $\lambda_{1}>0$ is the unstable pole and $0>\lambda_{2} \geq \lambda_{3} \geq \ldots \geq$ $\lambda_{m}$ are the real parts of the stable poles, where $m \leq n$. Conjugate complex pole pairs (i.e. $\lambda_{r} \pm j \mu_{r}$ ) are represented as square submatrices, where $\nu_{r} \in \mathbb{R}$ can by an arbitrary chosen value. Furthermore, upon state transformation,

$$
b=\left[\begin{array}{lllllll}
\lambda_{1} & \lambda_{2} & \cdots & b_{r_{1}} & b_{r_{2}} & \cdots & \lambda_{m}
\end{array}\right]^{T},
$$

where $b_{r_{1}}, b_{r_{2}}$ and also $\nu_{r}$ are given by imposing $A^{-1} b=$ $\left[\begin{array}{llll}1 & 1 & \ldots & 1\end{array}\right]$.

Assume that there exists an algebraic state reconstruction (supposition valid for TCV tokamak). Thus, we can replace the vertical controller (VS controller of Fig. 1a)) by a linear 
state feedback controller

$$
v=f x
$$

with a state reconstruction block (Fig. 1b)).

The saturation function is defined by

$$
u=\operatorname{sat}(v)= \begin{cases}-1 & \text { if } \quad v<-1 \\ v & \text { if } \quad-1 \leq v \leq 1 \\ 1 & \text { if } \quad v>1\end{cases}
$$

\section{B. ELM perturbation}

The shape of the model of an ELM perturbation is illustrated in Fig. 3. The perturbation starts at $t_{0}$, reaches its maximum at $t_{1}$ and vanishes at $t_{2}$. For our purposes, we only use the derivative $\dot{w}$ (see (1)) which is a piecewise constant signal.

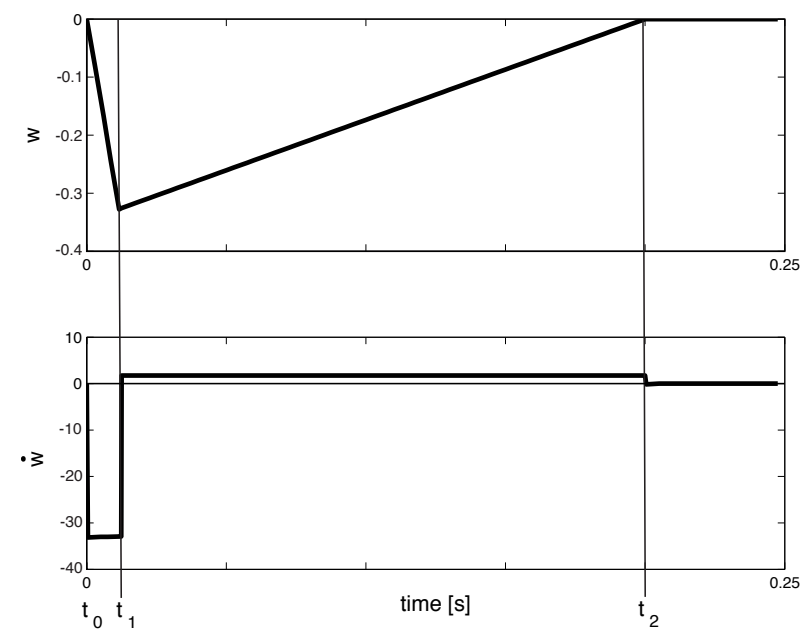

Fig. 3. Evolution of an ELM perturbation $w$ and its derivative $\dot{w}$.

\section{REVIEW OF THE RESULTS FOR SECOND ORDER SYSTEMS}

Consider a single input second-order linear system with an unstable $\left(\lambda_{1}>0\right)$ and a stable pole $\left(\lambda_{2}<0\right)$

$$
\dot{x}=A x+b u=\left[\begin{array}{cc}
\lambda_{1} & 0 \\
0 & \lambda_{2}
\end{array}\right] x+\left[\begin{array}{c}
\lambda_{1} \\
\lambda_{2}
\end{array}\right] u \text {. }
$$

With saturated linear state feedback, the closed-loop system is

$$
\dot{x}=A x+b \operatorname{sat}(f x),
$$

where $f$ is the feedback gain vector. The matrix $(A+b f)$ is assumed to be Hurwitz, i.e. the system is stable without saturation. Let $\tilde{\lambda}_{1}$ and $\tilde{\lambda}_{2}$ be the eigenvalues of $(A+b f)$. The two conditions that correspond to $(A+b f)$ being Hurwitz are: (i) $\lambda_{1}\left(1+f_{1}\right)+\lambda_{2}\left(1+f_{2}\right)<0$, and (ii) $\lambda_{1} \lambda_{2}\left(1+f_{1}+f_{2}\right)>0$. Since $\lambda_{1} \lambda_{2}<0$, the second condition gives $\left(1+f_{1}+f_{2}\right)<0$. Also, it can be verified that $f_{1}<0$, though $f_{2}$ can take either sign.

\section{A. The null controllable region and the region of attraction}

For a system with a single saturated input defined by (4) the set of admissible control is given by

$$
\mathcal{U}_{a}=[-1,1]
$$

Definition 1 Let $\Phi\left(t, x_{0}\right)$ denote the state of (5) at time $t$, starting with the initial condition $x_{0}$ at $t=0$. A state $x$ is said to be null controllable if there exists an admissible control $u(t) \in \mathcal{U}_{a}$ that steers the trajectory $\Phi(\cdot, x)$ to the origin

$$
\lim _{t \rightarrow \infty} \Phi(t, x)=0 .
$$

All states being null controllable belong to the set of the null controllable region which is denoted by $\mathcal{C}$.

The null controllable region for system (5) with input saturation (4) is given by ([1], [5], [6], [7])

$$
\mathcal{C}=\left\{x:\left|x_{1}\right|<1\right\}
$$

and its boundaries are defined by

$$
\partial \mathcal{C}_{+}=\left\{x: x_{1}=1\right\}, \partial \mathcal{C}_{-}=\left\{x: x_{1}=-1\right\} \text {. }
$$

Definition 2 Let $\Phi\left(t, x_{0}\right)$ denote the state of (6) at time $t$, starting with the initial condition $x_{0}$ at $t=0$. The region of attraction of the stable equilibrium point is defined by:

$$
\mathcal{A}=\left\{x: \lim _{t \rightarrow \infty} \Phi(t, x)=0\right\} .
$$

The boundary of $\mathcal{A}$ is denoted by $\partial \mathcal{A}$.

\section{B. The shape of the region of attraction}

It was shown in [1], [7] that if $f_{2}=0$ and $1+f_{1}<0$ is satisfied then $\mathcal{A}=\mathcal{C}$. For all other cases, where $f_{2} \neq 0$, the region of attraction is always strictly smaller than the null controllable region $(\mathcal{A} \subset \mathcal{C})$. The exact shape of the region of attraction of such systems is discussed in [4]. It results that the shape of the region of attraction $\mathcal{A}$ can be either: i) unbounded hyperbolically shaped or ii) a bounded limit cycle, depending on the parameters of the system and the controller. Fig. 4 shows the two different shapes for the same open loop system with different controller parameters $f_{1}$ and $f_{2}$, respectively: a) unbounded hyperbolically shaped and b) bounded limit cycle.

\section{Enlarging the region of attraction with a nonlinear controller}

Recent work has shown that it is possible to enlarge the region of attraction to include the full null controllable region $\mathcal{A}=\mathcal{C}$, without loss of local performance, by introducing a continuous nonlinear function in the controller [3]. Consider the controller

$$
v(x)=f_{1} x_{1}+k(x) f_{2} x_{2} \quad u=\operatorname{sat}(v),
$$




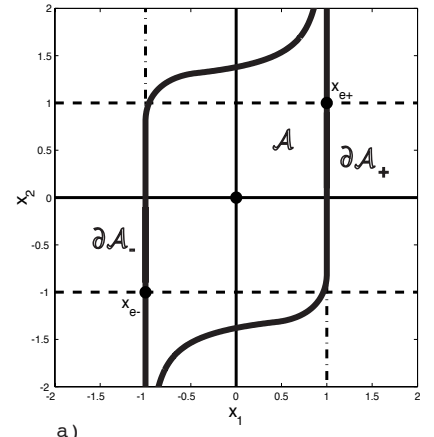

a)

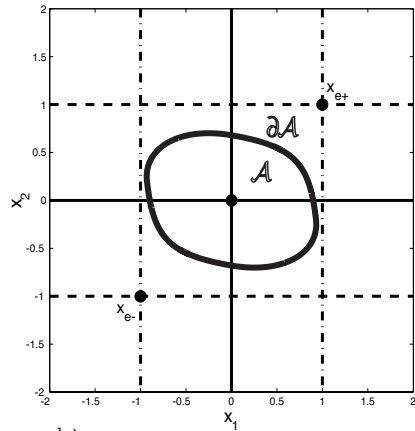

b)

Fig. 4. Different shapes of the region of attraction $\mathcal{A}$ for different controller parameters $f_{1}$ and $f_{2}$. a) unbounded hyperbolically shaped. b) bounded limit cycle.

where $f=\left[\begin{array}{ll}f_{1} & f_{2}\end{array}\right] \in \mathbb{R}^{2}$ and $k(x): \mathbb{R}^{2} \rightarrow \mathbb{R}$. Assume that $f$ has been chosen to get the desired performance of the closed-loop system near the origin. Compared to (6), the new controller (11) differs by the introduction of a continuous nonlinearity by choosing:

$$
k(x)=1-\left|x_{1}\right|,
$$

where $0<k(x) \leq 1$ since $\left|x_{1}\right|<1$ within the null controllable region.

The idea behind this nonlinear controller is as follows. If $x_{1} \approx 0$, then $k(x) \approx 1$ which implies that the controller is approximately the linear state feedback controller $v \approx$ $f_{1} x_{1}+f_{2} x_{2}$. In this case, the controller concentrates on local performance. On the contrary, if the unstable state approaches the boundary of the null controllable region $\mathcal{C}$, then $x_{1} \approx \pm 1$ and $k(x) \approx 0$. This implies that the controller is approximately the linear state feedback $v \approx f_{1} x_{1}$, where it focuses on the stabilisation of the unstable state and global stability $(\mathcal{A}=\mathcal{C})$. Moreover, since the controller (11)-(12) is a continuous one, chattering is avoided.

\section{CONTINUOUS NONLINEAR CONTROLLER FOR HIGHER ORDER SYSTEMS}

We consider the high order dynamic system (1) (order $n>$ 2 ) and its controller (3). Consider system (1) as a system with an anti-stable and a stable subsystem [5]

$$
\left[\begin{array}{l}
\dot{x_{1}} \\
\dot{x_{s}}
\end{array}\right]=\left[\begin{array}{cc}
\lambda_{1} & 0 \\
0 & A_{s}
\end{array}\right]\left[\begin{array}{l}
x_{1} \\
x_{s}
\end{array}\right]+\left[\begin{array}{l}
\lambda_{1} \\
b_{s}
\end{array}\right] u,
$$

where $x_{1} \in \mathbb{R}$ and $\lambda_{1}$ describe the anti-stable subsystem and $x_{s}=\left[\begin{array}{llll}x_{2} & x_{3} & \ldots & x_{n}\end{array}\right]^{T} \in \mathbb{R}^{n-1}, A_{s}$ and $b_{s}$ describe the stable subsystem. Thus, the null controllable region of system (1) is given by

$$
\mathcal{C}=\left\{x \in \mathbb{R}^{n}:\left|x_{1}\right|<1\right\} .
$$

This shows that the boundaries of the null controllable region are the hyperplanes

$$
\partial \mathcal{C}_{+}=\left\{x \in \mathbb{R}^{n}: x_{1}=1\right\}, \partial \mathcal{C}_{-}=\left\{x \in \mathbb{R}^{n}: x_{1}=-1\right\} \text {. }
$$

Therefore, the null controllable region is only restricted by the unstable state (anti-stable system) while the stable states (stable subsystem) can take any arbitrary values.

The next question is whether a linear controller exists for which the region of attraction is equal to the null controllable region $(\mathcal{A}=\mathcal{C})$. Consider the linear controller

$$
v(x)=f_{1} x_{1}+f_{2} x_{2}+f_{3} x_{3}+\ldots+f_{n} x_{n} .
$$

Then it can be shown that $\mathcal{A}=\mathcal{C}$, if and only if $f_{2}=f_{3}=$ $\ldots=f_{n}=0$. With such a choice of parameters the antistable subsystem becomes

$$
\dot{x_{1}}=\lambda_{1}\left(x_{1}+\operatorname{sat}\left(f_{1} x_{1}\right)\right) .
$$

Since the Hurwitz condition calls for $1+f_{1}<0$, then

$$
\begin{gathered}
\dot{x_{1}}<0 \forall x_{1}>0, \quad \dot{x_{1}}>0 \forall x_{1}<0 \quad \text { and } \\
\dot{x_{1}}=0 \text { for } x_{1}=0
\end{gathered}
$$

is satisfied for $x_{1} \in \mathcal{C}$ leading the unstable state $x_{1}$ to converge to zero independently of the other states. Since $x_{1} \rightarrow 0$, this implies that $u \rightarrow 0$ and the stable subsystem becomes $\dot{x_{s}}=A_{s} x_{s}$. Therefore, since $A_{s}$ is stable, $x_{s} \rightarrow 0$. For all other linear controllers where at least one of the parameters $f_{2}, f_{3} \ldots f_{n}$ is nonzero, $\mathcal{A} \subset \mathcal{C}$.

In the extension of the nonlinear controller (11) to higher order systems, the idea is to push all the terms except $f_{1} x_{1}$ to zero as $\left|x_{1}\right| \rightarrow 1$. This is done by the following modification:

$$
v(x)=f_{1} x_{1}+k(x)\left(f_{2} x_{2}+f_{3} x_{3}+\ldots+f_{n} x_{n}\right)
$$

and

$$
k(x)=1-\left|x_{1}\right| \quad \text { if }\left|x_{1}\right| \leq 1 .
$$

The reason for using the same $k(x)$ to push each of the terms $f_{2} x_{2}, f_{3} x_{3}, \ldots, f_{n} x_{n}$ to zero is due to the fact that the system without saturation and with a constant $k(x) \in[0,1]$ can be proven to be stable. Of course this proposition does not constitute a proof of the global stability of the closed loop system with saturation. But it is an interesting property encouraging the research of a formal stability proof. Another promising fact is that all simulations done until now have revealed to be stable.

Proposition 1 Consider (13) along (15)

$$
u(x)=f_{1} x_{1}+\bar{k}\left(f_{2} x_{2}+f_{3} x_{3}+\ldots+f_{n} x_{n}\right)
$$

where $\bar{k} \in[0,1]$ is fixed. If $1+f_{1}<0$ and $A+b f$ is Hurwitz then the feedback system is stable for any $0 \leq \bar{k} \leq 1$.

Proof: Consider only the feedback $u=f_{1} x_{1}$. This leads to the closed loop state matrix

$$
\bar{A}=A+b f_{1} x_{1}=\left[\begin{array}{cc}
\lambda_{1}\left(1+f_{1}\right) & 0 \\
b_{s} f_{1} & A_{s}
\end{array}\right] .
$$

By construction $\bar{A}$ is stable.

Now, consider the input-output system

$$
\begin{gathered}
\dot{x}=\bar{A} x+b \bar{u} \\
\bar{y}=-\bar{f} x
\end{gathered}
$$


where $\bar{f}=\left[\begin{array}{lllll}0 & f_{2} & f_{3} & \ldots & f_{n}\end{array}\right]$. This system is open loop stable since $\bar{A}$ is stable. Let the open-loop transfer function of (19) be denoted by

$$
\frac{\bar{Y}(s)}{\bar{U}(s)}=L(s)
$$

Consider the closed-loop system

$$
\dot{x}=\bar{A} x+b \bar{f} \bar{k} x=A x+b u(x)
$$

which can be considered as the closed loop system with $L(s)$ and $\bar{k}$ in the loop. Since $L(s)$ is stable and the closed-loop system is stable with $\bar{k}=1$ (this is due to $A+b f$ being Hurwitz) the Nyquist plot of $L(s)$ does not encircle $(-1,0)$ in the complex plane. Thus, there exists no $\omega \in \mathbb{R}$ for which following both conditions are satisfied

$$
|L(j \omega)|>1 \quad \text { and } \quad \angle L(j \omega)= \pm \pi .
$$

It can be seen that for $0 \leq \bar{k} \leq 1$ we have $|\bar{k} L(j \omega)| \leq$ $\bar{k}|L(j \omega)| \leq|L(j \omega)|$ and $\angle(\bar{k} L(j \omega))=\angle L(j \omega)$. Thus, from (22) there exists no $\omega \in \mathbb{R}$ for which both conditions

$$
|\bar{k} L(j \omega)|>1 \text { and } \angle(\bar{k} L(j \omega))= \pm \pi
$$

are satisfied implying that the Nyquist plot of $\bar{k} L(s)$ does not encircle $(-1,0)$. Therefore, since $\bar{k} L(s)$ is stable the closedloop system (21) is stable for every $0 \leq \bar{k} \leq 1$.

\section{A. Limited duration perturbation}

In general, when the state leaves the null controllable region there is no possibility of bringing it back to the origin by only using the control $u$. However, when faced with perturbations such as that shown in Fig. 3, the positive slope of the perturbation can help the system return to the null controllable region and then subsequently to the origin. In such a case the control action outside the null controllable region becomes equally important. For this, the control law

$$
k(x)=0 \quad \text { if }\left|x_{1}\right|>1 .
$$

has to be added to ensure that only the unstable state is fed back when it is outside the null controllable region.

Remark: The reference controller is implemented as an input-output controller, while the proposed one is a state feedback controller. The latter calls for state reconstruction which might be interpreted as a drawback. However, since the boundaries of the null controllable region are only determined by the unstable state $\left|x_{1}\right|<1$ (8), it is not possible to enlarge the region of attraction to the null controllable region without this knowledge of $x_{1}$.

\section{COMPARISON OF CONTROLLERS VIA SIMULATIONS}

In this section we compare via simulation the reference controller, given by $v=f x$, against the new continuous nonlinear controller given by (15), (16) and (24). The comparison is illustrated in phase diagrams. Since we deal with a high order system (50 .. 100 states) we cannot show the

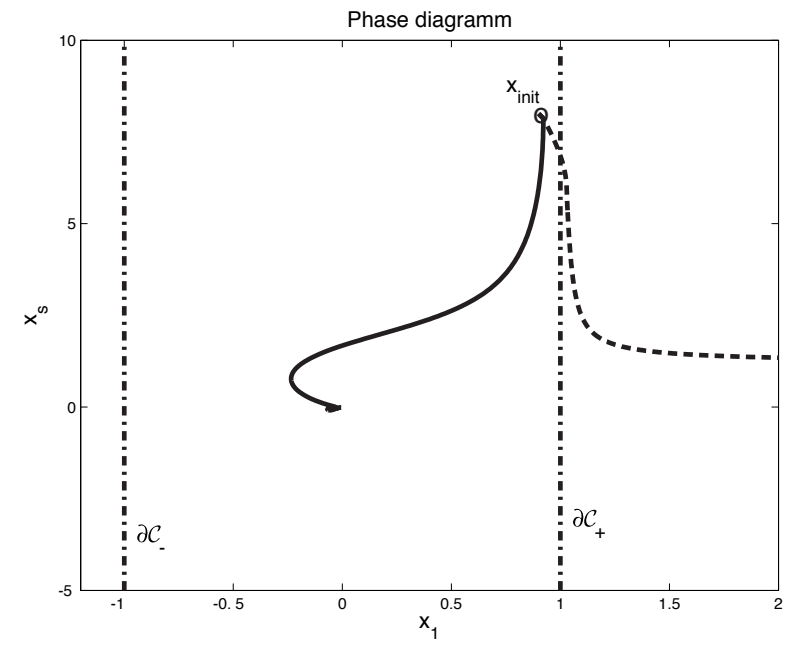

Fig. 5. Example with non-zero initial conditions $\left(x_{i n i t}\right)$ and without perturbation, dashed: reference controller, solid: continuous nonlinear controller.

evolution of all states. Thus, the phase diagrams show the evolution of only two states: i) the unstable state, denoted by $x_{1}$ and ii) one of the most disturbed stable states, denoted by $x_{s}$. For what follows, the region of attraction of the reference controller is denoted by $\mathcal{A}_{n}$ and the region of attraction of the continuous nonlinear controller is denoted by $\mathcal{A}_{c}$. We show via simulation that for the reference controller the region of attraction is strictly a subset of the null controllable region $\mathcal{A}_{n} \subset \mathcal{C}$. Furthermore, we show that the trajectories of the closed-loop system with the nonlinear controller converge to the origin if the initial conditions are inside $\mathcal{A}_{c}=\mathcal{C}$.

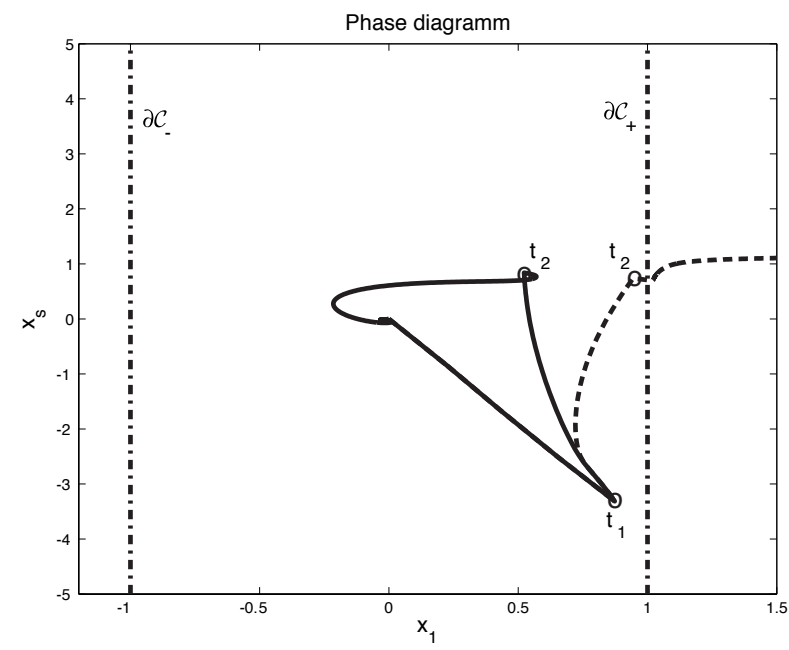

Fig. 6. Example with a large perturbation, dashed: reference controller, solid: continuous nonlinear controller.

To disturb the system away from the equilibrium we apply an ELM perturbation as illustrated in Fig. 3. The perturbation starts at $t_{0}$, reaches its maximum at $t_{1}$ and vanishes at $t_{2}$. 
Since it is difficult to know whether the state remains in the region of attraction during the perturbation, we have to wait until the perturbation vanishes at $t=t_{2}$ to determine if the controller is able to stabilise the system.

\section{A. Initial conditions}

For the first example we do not disturb the system, thus $\dot{w}=0$. Instead, we set non-zero initial conditions. The phase diagram (Fig. 5) shows the evolution of the unstable state, denoted by $x_{1}$ and one of the most disturbed stable states, denoted by $x_{s}$. The point $x_{i n i t}$ denotes the initial conditions which are located inside the null controllable region $x_{\text {init }} \in$ $\mathcal{C}$. Since for the nonlinear controller the initial conditions are located in the region of attraction $x_{\text {init }} \in \mathcal{A}_{c}=\mathcal{C}$ the trajectory converges to the origin. For the reference controller the trajectory diverges, thus confirming by simulation that $\mathcal{A}_{n} \subset \mathcal{C}$.

\section{B. Large perturbation}

The second example shows the evolution of the trajectories for both controllers during and after a large perturbation (Fig. 6). At $t_{2}$ the states of the systems with both controller are in $\mathcal{C}$. Since for the nonlinear controller $\mathcal{A}_{c}=\mathcal{C}$, the trajectory converges to the origin. For the reference controller the trajectory diverges and thus, the state is not in $\mathcal{A}_{n}$.

\section{Huge perturbation}

The third example shows the trajectory evolutions for a much larger perturbation amplitude (Fig. 7). Both trajectories leave the null controllable region $\mathcal{C}$ and only the trajectory for the system with the nonlinear controller reenters $\mathcal{C}$. Therefore, this trajectory converges to the origin and the trajectory of the system with the reference controller diverges.

For all these examples, the unstable state $x_{1}$ is brought back to the origin faster when the continuous nonlinear controller is used. This is the benefit of the nonlinear function $k(x)$ which helps the controller concentrate on the unstable state in the proximity of the boundaries of $\mathcal{C}$ and beyond it.

\section{CONCLUSION}

In this paper, a simple continuous nonlinear controller for the stabilisation of the ITER tokamak unstable vertical position in the presence of actuator saturation is proposed. The main idea was to modify an existing linear controller by introducing a nonlinear term to the control law. This new controller enlarges the region of attraction to the maximal reachable region of attraction under input saturation which is the null controllable region. Additionally, its local performance around the origin is similar to that of the existing linear controller. An additional advantage of the nonlinear controller is that the unstable state is brought back faster to the origin and thus, the rejection of the perturbation is more efficient. This is the benefit of the nonlinear function where

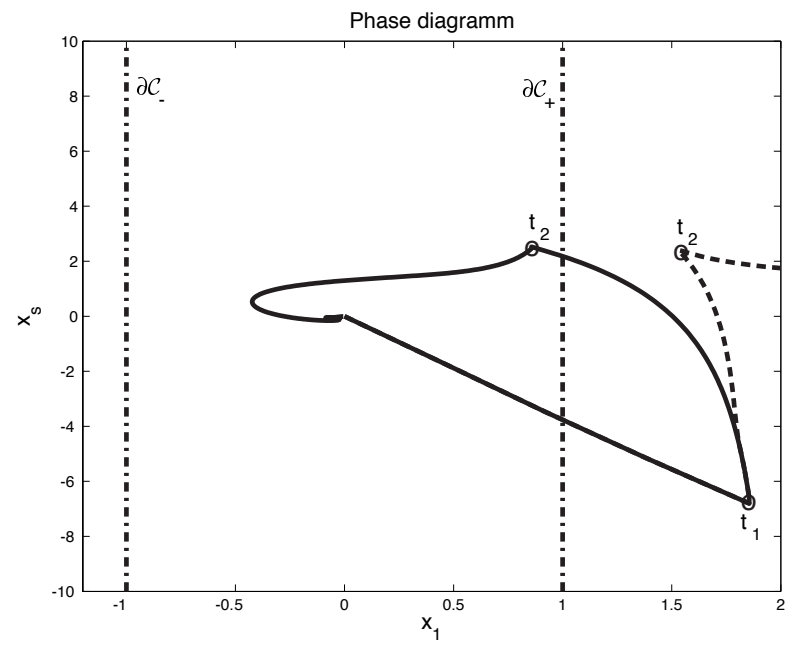

Fig. 7. Example with a huge perturbation, dashed: reference controller, solid: continuous nonlinear controller.

the controller concentrates on the control of the unstable state in the proximity of the boundaries of null controllable region and beyond it.

\section{REFERENCES}

[1] J. Alvarez, R. Suárez, and J. Alvarez. Planar linear systems with single saturated feedback. System \& Control Letters, 20:319-326, 1993.

[2] M. Ariola, A. Pironti, and A. Portone. Vertical stabilization and plasma shape control in the iter-feat tokamak. In Proceedings of the 2000 IEEE International Conference on Control Applications, pages 401-405, Anchorage, Alaska, USA, 2000.

[3] J-Y. Favez, Ph. Mullhaupt, B. Srinivasan, and D. Bonvin. A globally stabilising controller under saturated input for linear planar systems with one unstable pole. In Submitted to American Control Conference ACC 2004, Boston, 2004.

[4] J-Y. Favez, B. Srinivasan, Ph. Mullhaupt, and D. Bonvin. Condition for bifurcation of the region of attraction in linear planar systems with saturated linear feedback. In $41^{\text {th }}$ Conference on Decision and Control, pages 39183923, Las Vegas, USA, 2002.

[5] T. Hu and Z. Lin. Control Systems with Actuator Saturation: Analyses and Design. Birkhauser, Boston, 2000.

[6] T. Hu, Z. Lin, and L. Qiu. Stabilization of exponentially unstable linear systems with saturating actuators. IEEE Transactions on Automatic Control, 46(6):973979, 2001.

[7] L. Scibile and B. Kouvaritakis. Stability region for a class of open-loop unstable linear systems: theory and application. Automatica, 36:37-44, 2000. 